\title{
HOW DO NEUTRON STAR RADII DEPEND ON THEIR CORE-CRUST EOS?
}

\author{
Chikako Ishizuka* \\ Faculty of Science and Technology, Tokyo University of Science, 2641 Yamazaki, Noda, Chiba, \\ 278-8510 Japan \\ E-mail: Chikakodrs.tus.ac.jp \\ Ken'ichiro Nakazato \\ Faculty of Science and Technology, Tokyo University of Science, 2641 Yamazaki, Noda, Chiba, \\ 278-8510, Japan \\ E-mail: nakazatodrs.tus.ac.jp
}

\section{Akira Ohnishi}

Yukawa Institute for Theoretical Physics, Kyoto University, Kitashirakawa Oiwakecho,

Sakyo-ku, Kyoto 606-8502, Japan

E-mail: hnishidyukawa.kyoto-u.ac.jp

\begin{abstract}
Nuclear equation of state $(\mathrm{EoS})$ provides the fundamental property of nuclear many body systems. It is expected to be constrained by ongoing astronomical observations of the mass and radius of neutron stars (NSs). It is known that even the same nuclear EoS gives different behaviors for NS radii on the mass-radius plane depending on the treatment of connecting the EoSs at the corecrust boundary. However, there are no systematic surveys on this issue. We report the results of systematic surveys on NS radii by assuming that NS models consist of crust EoSs and core nuclear EoSs. We adopt a variety of EoSs in the literature for crust EoSs, and various parameters of relativistic mean field for core nuclear EoSs. The dependence of NS radii on the core-crust boundary is investigated by solving the Tolman-Oppenheimer-Volkoff equation by taking account the following cases of the boundary. Case-I: the boundary density is the highest density in a given data set for the crust. Case-II: the boundary density exist at somewhere in the sub-nuclear region. In this case, we examine the influence of the crust varying the boundary from 0.1 to 1.0 times the nuclear saturation density $\left(\rho_{0}\right)$ in steps of $0.1 \rho_{0}$.
\end{abstract}

XIII International Symposium on Nuclei in the Cosmos

July 7-11, 2014

Debrecen, Hungary

\footnotetext{
* Speaker.
} 


\section{Introduction}

Constraint on nuclear models using the observations of neutron stars has been investigated for decades. Massive neutron stars of two solar masses within small observational error-bars, have attracted much attention to the constraints. Added to that, the NICER, LOFT and Astro-H are expected to succeed in a simultaneous observation of mass and radius for a neutron star. Observational efforts to determine a neutron star radius $R_{N S}$ with $\sim 0.5 \mathrm{~km}$ accuracy presume that the ambiguity of theoretical models must be smaller than observational constraints. However, this is not necessarily guaranteed. A model of the equation of state for NS crust region is not always the same as that for NS core. In the crust, light nuclei such as alpha are abundant, so that a nuclear model, which can describe clustering feature, may be favoured. On the other hand, in the core, a model, which includes enough many-body effect, may be favoured. Then if the best morel for the crust and that for the core are different, what happens?

In general, we compare the lines of models and parameters without error-bars in the $M R$-plane, in order to constrain the equation of state using the theoretical solution of the TOV equation, not so much attention has been paid to the contribution to a radius from the crust phase. However, the NS radius can vary for different component of neutron star matter by the order of a $\mathrm{km}$. As mentioned before, the consistency between the crust and the core models is not necessary. It allows us various ambiguity in the combination of models for both phases and in the treatment of their boundary.

These ambiguity can be no longer negligible, because the accuracy of a NS radius becomes comparable to the difference between theoretical models. Actually, in NICER project of ESA, the observational ambiguity is suppressed around $\sim 0.5 \mathrm{~km}$. Such progress can be realized by very high energy-resolution by overcoming technical difficulty. In this situation, if the treatment of a crust gives its radius large ambiguity, we cannot constraint nuclear models as accurate as expected even with the best possible observations.

In this study, we systematically investigate the influence of core-crust EoSs on $R_{N S}$ for isolated cold NS without rotation, because the radius is related with the EoS around $\rho_{0}$. Our purpose is to perform a quantitive evaluation of the theoretical ambiguity brought by the treatment of the crust phase, by using representative data set of the crust EoSs. We describe the treatment of crust EoSs in section 2, evaluate its contribution to a NS radius and investigate the background physics in section 3 , and summarize our results in section 4 .

\section{Set up}

We solve the Tolman-Oppenheimer-Volkoff (TOV) equation,

$$
\frac{d P(r)}{d r}=-\frac{G}{r^{2}}\left(\rho(r)+\frac{P(r)}{c^{2}}\right)\left(M(r)+4 \pi r \frac{P(r)}{c^{2}}\right)\left(1-\frac{2 G M(r)}{c^{2} r}\right)^{-1},
$$

using various sets of nuclear EoSs for core and crust region. Connection of core and crust EoS is as follows: (Step-1) Smooth connection using cubic spline interpolation avoiding negative-gradient pressure. (Step-2) Simple connection between crust and core tables. For the crust and core EoSs, we selected representative EoSs which are open to the public.

In Table [, combinations of the crust and core EoSs are shown. The figures inside the parenthesis in the first column reveals the densities which are covered in each data. Here they are normalized 
Table 1: The list of NS crust and core EoSs used in our calculation. For the crust EoSs, the densities included in each table are shown.

\begin{tabular}{l|l}
\hline Crust EoS (density region) & Core EoS (parameter set) \\
\hline $\operatorname{MYN}\left(\rho_{B}<\rho_{0}\right)$ & RHF(MYN) \\
$\operatorname{BBP}\left(\rho_{B}<0.8 \rho_{0}\right)$ & RMF(TM1) \\
NGB $\left(\rho_{B}<0.5 \rho_{0}\right)$ & Ab initio (FPS) \\
HZ $\left(\rho_{B}<0.5 \rho_{0}\right)$ & Skyrme HF (SLy) \\
\hline
\end{tabular}

by the standard nuclear density, $\rho_{0}$. We find that the most of the upper limit of these data are lower than $\rho_{0}$. It means that these tables automatically determine the boundaries between core and crust, regardless of physical correctness of these boundary. In order to avoid the unrealistic boundary, the consistent treatment of the crust and core might be important. The second column denotes the adopted framework. The acronyms "RHF”, “RMF”, “Ab initio”, and "Skyrme HF” mean relativistic Hartree-Fock, relativistic mean field, ab initio variational method, and Skyrme HartreeFock, respectively. The label inside the parenthesis shows the parameter set or the representative calculation of the adopted theoretical framework.

It is natural that the EoS of the crust is different from the core as we mentioned in the previous section. We assume that the boundary is not given by the table, but by the physical condition. The boundary varies by $0.1 \rho_{0}$ for $(0.1-1.0) \times \rho_{0}$, because the boundary should be below the standard saturation density and sub-nuclear density. Note that we cannot always use every boundary density between $(0.1-1.0) \times \rho_{0}$ in all combinations, due to the upper limit in each data table. We expect that the physical boundary density will be determined by the future nuclear experiments and astronomical observations. As for the connecting point of the crust and core, we smoothly connected two EoSs with cubic spline interpolation, avoiding the negative energy and pressure gradient to the density.

\section{Results \& Discussion}

Following figures show the $R_{N S}$-ambiguity about the treatment of core-crust boundary and crust models. Both selection of $\rho_{B N D}$ and treatment of the boundary affect $R_{N S}$. In this section, we show the contribution of the crust treatment (the selection of the boundary and the connecting method) to the radius of a neutron star. As seen in Figure 1, the crust contribution only appears in the radius. For the solution of the TOV equation, no effect was found in the mass. Figure 1 is the result of the case with the MYN EoS for the crust and the TM1 EoS for the core. In this combination, we can systematically see the influence of the crust for all the possible boundary below the saturation. This is the best combination to see the crust effect in Table 1. The red line in the most right side (a line with '(2)' in Fig. 2) is the mass and radius relation with the boundary, which is given by the upper end of the used data table. The lines inside the large circle (red line in the most left side (a line with '(1)') and dashed lines) are the case with the given boundary for $0.1 \rho_{0} \leq \rho_{B N D} \leq \rho_{0}$. 


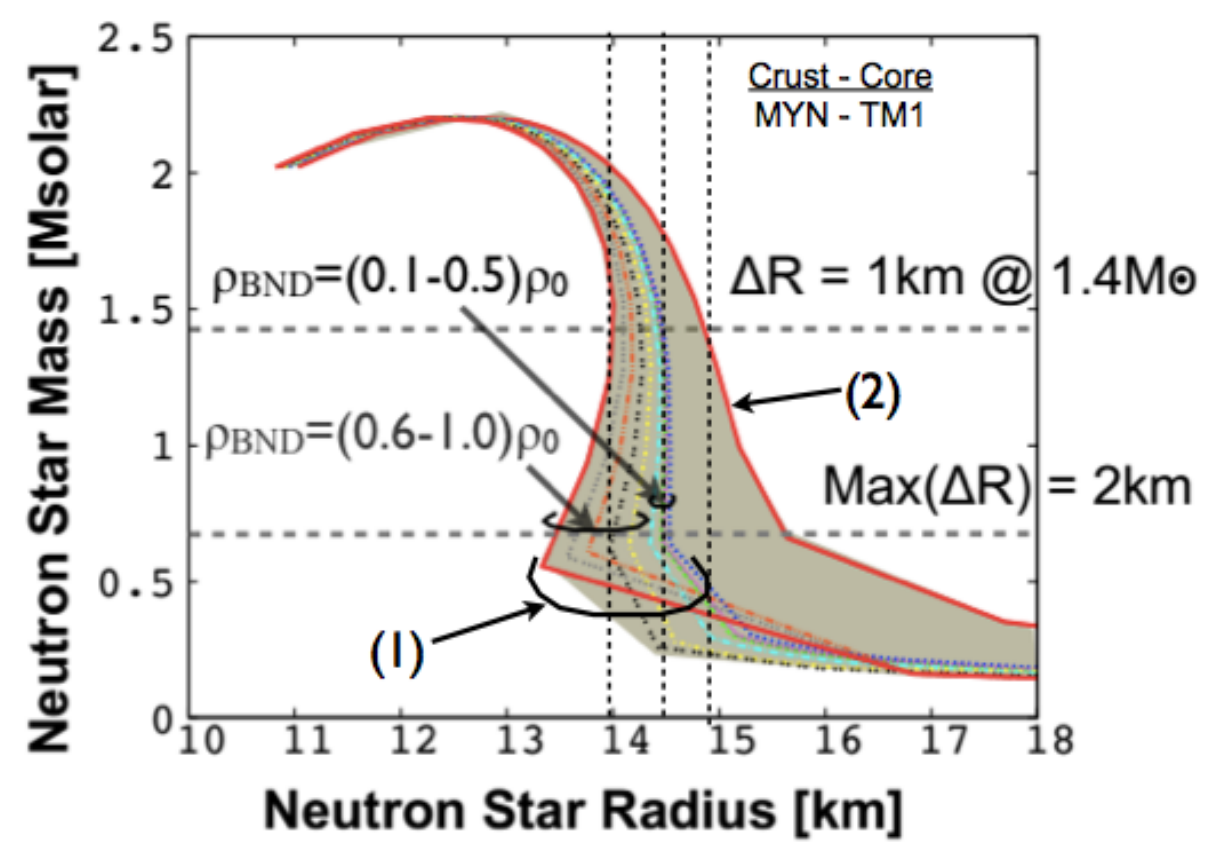

Figure 1: The mass and radius relations with various treatments of NS crust.

The M-R curve with the simple connection (the line (2)) shows the largest radii, while cases with the smooth connection (the lines (1)) are smaller than the case (2). We performed more detailed analysis for the group (1). We can divide the group (1) into two groups by the boundary density. One is the case with $\rho_{B N D}=(0.1-0.5) \times \rho_{0}$ and the other is that with $(0.6-1.0) \times \rho_{0}$. The former group with smaller boundary densities does not show the distinguished change in the radii due to the boundaries. On the contrary, the boundaries drastically affect the radii in the latter group with large boundary densities.

The ambiguity due to the treatment of the crust (a gap between the case (1) and (2)) in the sub-nuclear density region, is about $2 \mathrm{~km}$ at maximum, when the mass of the neutron star is about the half of the canonical mass. The ambiguity even at the canonical mass is still $1 \mathrm{~km}$ large. It is comparable with the difference between the nuclear models. These results suggest that the ambiguity of the crust treatment can not be negligible when the crust-core boundary is between $0.6 \rho_{0}$ and the saturation density.

Figure 2 shows the ambiguity caused by the core EoSs and the relative gap compared to the ambiguity by the crust EoSs using three sets of the combinations of the crust and core. We can see the difference of the core with a fixed crust EoS, and that of the crust with a fixed core EoS.

For the comparison of the crust, we used TM1 as a core, and BBP and MYN as a crust. For the comparison of the core, we used TM1 and MYN as a core, and MYN as a crust. In Fig.2, the gap between different core models is about $\Delta($ Core $) \simeq 1.5 \mathrm{~km}$. In the present case, we can constraint the core within the ambiguity of the crust. However, if the ambiguity caused by the core models is as large as that by the crust, it is difficult to constrain the nuclear models within the crust ambiguity. One should also note that the $\Delta R$ (Core) is comparable to ambiguity from the treatment of the boundary within $\Delta R($ Obs. $)$. 


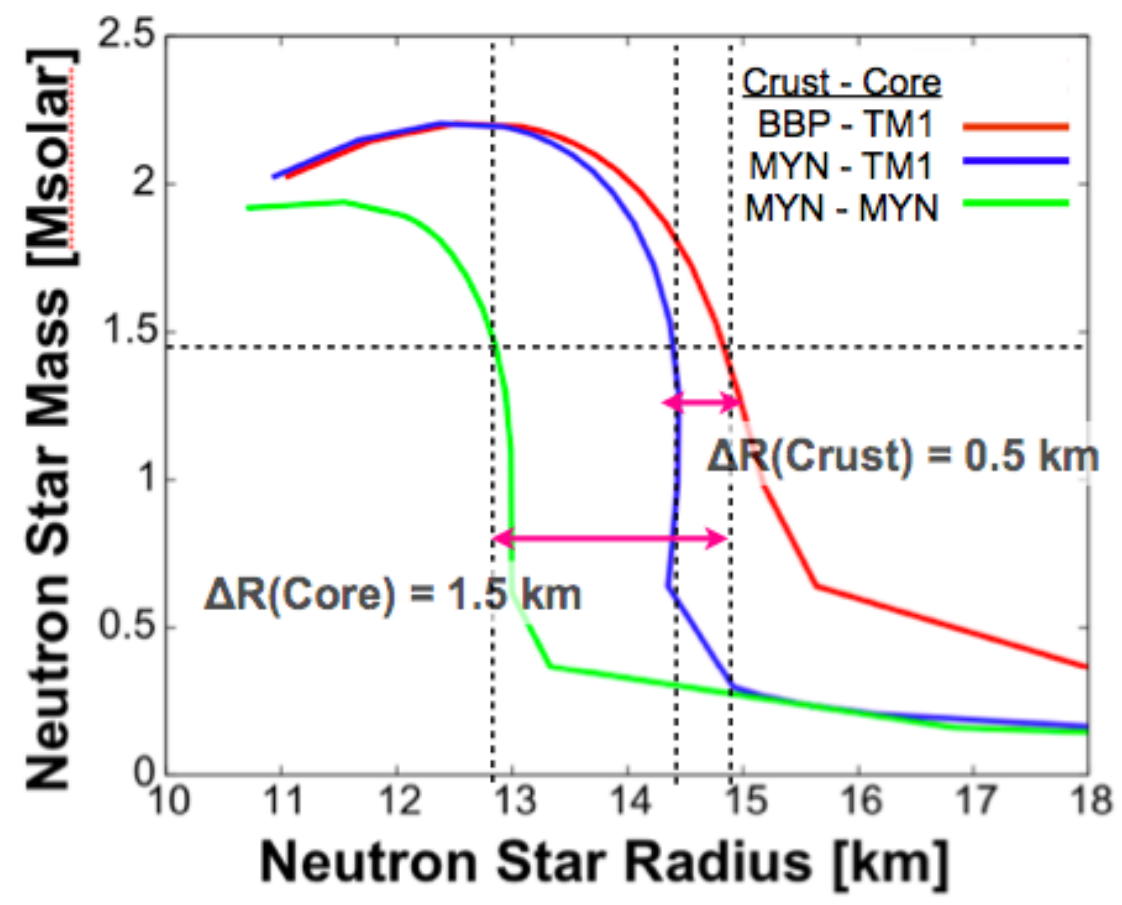

Figure 2: The mass and radius relation with fixed core and fixed crust.

For further discussion, we estimate the crust and core boundary as the region where nuclei exist and as region where no nuclei exist, respectively. Around the critical density of two groups inside the circle (1) in Fig.1, nuclear pasta phase or spinodal unstable region has been known as an inhomogeneous phase which contains nuclei. Note that the existence of the pasta phase in a neutron star is still under debate.

The pasta phase has been studied by various schemes. Among them, the current consideration is based on the model of Oyamatsu \& Iida (2007), which discuss the pasta phase with a parameter relating to the nuclear symmetric energy. Within observational and experimental constraints on $L(30<L / M e V<110)$, nuclear pasta can exist around $\rho_{B}<0.5 \rho_{0}[9]$ in the crust. It means $\rho_{B N D} \sim$ $0.5 \rho_{0}$ at highest with pasta phase. Then we can find that the ambiguity of the crust can be negligible.

On the other hand, if the pasta phase does not appear in a neutron star, the spinodal region under the $\beta$-equilibrium should be used as the crust boundary. Figure 3 shows the spinodal unstable phase under the $\beta$-equilibrium with a typical lepton to baryon ratio $Y_{L}=0.4$ in the case of the parameter set TM1 of the relativistic mean field. From Fig. 3, if pasta phase does not exist in the crust, the core-crust boundary will be determined by the edge of spinodal region. In the NS matter at $\mathrm{T}=0 \mathrm{MeV}$, the boundary density will be $\rho_{B N D} \sim 0.8 \rho_{0}$ at highest [10]. It means that we cannot neglect the crust ambiguity to the radius, as mentioned in Fig. 2.

\section{Summary and future prospects}

The RNS gives us better constraints on EoS models than $M_{N S}$. In this study, we investigated the influence of the core-crust boundary on $R_{N S}$, compared with the observational ambiguity. We 


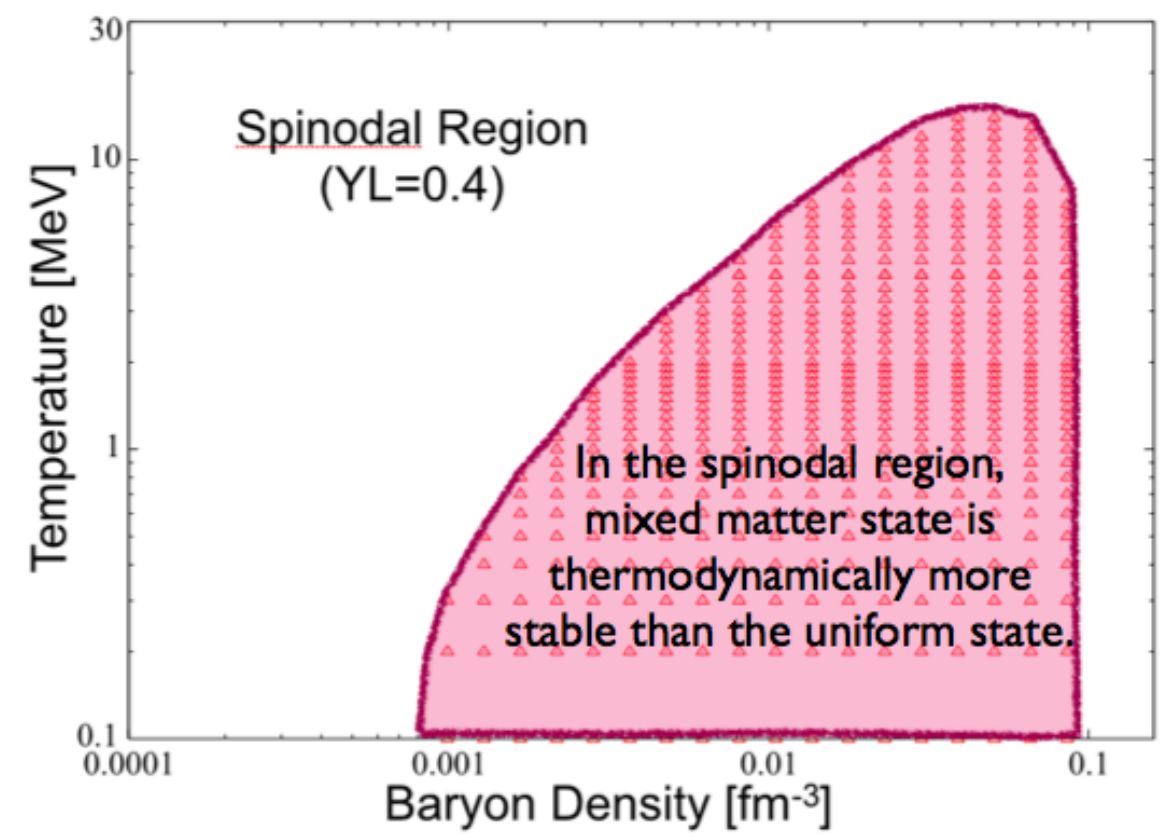

Figure 3: The spinodal unstable region with the lepton to baryon ratio $Y_{L}=0.4$ for neutron star matter.

also systematically studied the ambiguity of the crust EoSs.

As a result, we find the followings: Most of the existent crust EoSs contain data only for $\rho_{B}<0.5 \rho_{0}$. Obtained $\Delta R$ could be as large as difference among EoS models. The $\Delta R($ Crust $)$ can be removed by crust-oscillation [8]. As the criterion to select $\rho_{B N D}$, we use nuclear pasta and spinodal phase. The magnitude of $\Delta R$ depends on the criterion.

\section{References}

[1] C. Ishizuka et al., in prep.

[2] T. Miyatsu et al., Astrophys. J 777, 4 (2013).

[3] Y. Sugahara \& H. Toki, Nucl. Phys. A 579, 557 (1994).

[4] SLy and FPS are from the formula given in P. Haensel \& A. Y. Potenkin, Astron. and Astophys. 428, 191 (2004).

[5] G. Baym et al., Nucl. Phys. A 175, 225 (1971).

[6] W. G. Newton et al., Astrophys. J. Suppl. 204, 9 (2013).

[7] P. Haensel \& J. L. Zdunik, Astron. and Astrophys. 480, 459 (2008).

[8] H. Sotani et al., Monthly Nortice of Roy. Astron. Soc. 434, 2060 (2013).

[9] K. Oyamatsu, \& K. Iida, Phys. Rev. C 75, 015801 (2007).

[10] C. Ishizuka, Nucl. Phys. A 723, 517 (2003). 